\title{
Correlation Of Sputum Gram's Stain And Culture In Lower Respiratory Tract Infections
}

\author{
Anuradha Mokkapati ${ }^{1}$, Madhavi Yalamanchili ${ }^{2}$ \\ ${ }^{1}$ MD, Associate Professor, Department of Microbiology, Apollo Institute of Medical Sciences and Research, \\ Jubilee Hills, Hyderabad- 96. \\ ${ }^{2}$ M.Sc Microbiology, Scientific officer, Super Religare Laboratories Limited, Hyderabad.
}

\begin{abstract}
Background: For diagnosis of Lower respiratory tract infections (LRTIs), expectorated sputum is the most commonly received sample in the laboratory. Normal resident bacteria of the oropharynx usually contaminate the sputum sample. The value of sputum microscopy and culture in the diagnosis, management and outcome of LRTIs is a matter of controversy. The following study was conducted to evaluate the correlation of Gram's stain and culture in sputum samples from LRTIs. Materials \& Methods: The present study was during a one year period (January to December 2012). A total of 120 sputum samples were processed. All the samples were processed through Gram's stain and culture. The Gram's smear was examined for the presence of polymorphs, epithelial cells and bacterial forms. Sputum samples were evaluated based on Bartlett's grading system. The cultures were incubated at $37^{\circ} \mathrm{C}$ overnight and identified in a Microscan autoSCAN 4 instrument (SIEMENS). Results And Conclusions: Out of 120 sputum samples processed, 78 (65\%) were acceptable and $42(35 \%)$ were not meeting the standard criteria of Bartlett. Potential pathogens were obtained from 70 of 78 acceptable samples (89.74\%), and from 4 of 42 non-acceptable samples (9.52\%). Gram's stain could detect similar morphological forms as that obtained in culture in 62 of the acceptable samples (sensitivity- 79.48\%) and 4 of the non acceptable samples (sensitivity -4.76\%). The most common organisms isolated in the present study include: Klebsiella pneumoniae- 22.85\%, Streptococcus pneumoniae- $11.49 \%$ and Staphylococcus aureus- $10 \%$. As authors of the present study, we recommend initial screening of sputum samples for clinically relevant results, and reject the non-acceptable samples, and re-order for fresh and correct specimens.
\end{abstract}

Key Words: Sputum, Gram's stain, Bartlett's criteria, sputum culture.

Accepted Date: 19 June 2013

\section{Introduction}

Lower respiratory tract infections (LRTIs) are a common cause of morbidity and mortality worldwide. For diagnosis of LRTIs, expectorated sputum is the most commonly received sample in the laboratory, which can be obtained easily and non-invasively. Normal resident bacteria of the oropharynx usually contaminate the sputum sample, and a large number of different species overgrow preventing the determination of the true pathogen[1]. Another commonly seen problem is that unless the patients are well trained, most of the times it is watery saliva which is sent instead of the purulent sputum to the laboratory, leading to erroneous results. The value of sputum microscopy and culture in the diagnosis, management and outcome of LRTIs is a matter of controversy. Traditional recommendations for management usually include Gram's stain and culture of sputum for routine evaluation. Some proponents feel that a properly performed sputum Gram's stain examined according to the correct guidelines is definitely useful in the initial evaluation of patients with pneumonia. Some others suggest that sputum Gram's stain and culture are neither sensitive nor specific for diagnosis of Community Acquired Pneumonia (CAP)[2]. With the above set back ground, the following study was conducted to evaluate the correlation of Gram's stain and culture in sputum samples from LRTIs.

\section{Materials And Methods}

The present study was during a one year period (January to December 2012). A total of 120 sputum samples were processed during the study period. All the 120 sputum samples were processed through Gram's stain and inoculation onto Blood agar, Chocolate agar and Mac Conkey agar. The Gram's smear was examined for the presence of polymorphs, epithelial cells and bacterial forms. Sputum samples were evaluated based on Bartlett's grading system and a score was given. 
2.1. Bartlett's CRITERIA[3] used:

\begin{tabular}{|l|l|}
\hline Number of Neutrophils /10 X LPF & GRADE \\
\hline$<10$ & 0 \\
\hline $10-25$ & +1 \\
\hline$>25$ & +2 \\
\hline Presence of mucus & +1 \\
\hline Number of Epithelial Cells /10 X LPF & \\
\hline $10-25$ & -1 \\
\hline$>25$ & -2 \\
\hline TOTAL SCORE & \\
\hline
\end{tabular}

Average number of epithelial cells and neutrophils in 20-30 LPFs was calculated and the total score arrived at. A final score of 0 or less indicated lack of active inflammation or contamination (non-acceptable sample), and a score of 1 and above was considered an acceptable sample. The inoculated culture plates were incubated overnight at $37^{\circ} \mathrm{C}$ in a carbondioxide incubator. A Gram's stain was done the next day from the growth and examined. The organisms were identified and antibiotic sensitivity done using Microscan autoSCAN-4 instrument (SIEMENS). Prompt Inoculation System D (SIEMENS) was used to prepare the inocula for biochemical reactions and antibiotic sensitivity testing. The prepared inocula were poured into either Gram positive or Gram negative panels depending on the morphology and staining characters of the isolates so obtained. The inoculated panels were incubated over night at $37^{\circ} \mathrm{C}$ in an incubator and read the next day in Micro SCAN auto SCAN-4 instrument for the final identification of the organism.

\section{Results}

Based on Bartlett's screening criteria, out of 120 sputum samples processed, 78 (65\%) were acceptable and $42(35 \%)$ were not meeting the standard criteria. Potential pathogens were obtained from 70 of 78 acceptable samples $(89.74 \%)$, and from 4 of 42 non-acceptable samples (9.52\%). Gram's stain could detect similar morphological forms as that obtained in culture in 62 of the acceptable samples (sensitivity- 79.48\%) and 4 of the non acceptable samples (sensitivity $-4.76 \%$ ).

\subsection{ISOLATES OBTAINED}

\begin{tabular}{|l|l|l|}
\hline ORGANISM & NO & $(\%)$ \\
\hline Klebsiella pneumoniae & 16 & 22.85 \\
\hline Streptococcus pneumoniae & 8 & 11.49 \\
\hline Staphylococcus aureus & 7 & 10 \\
\hline Haemophilus influenzae & 5 & 7.14 \\
\hline Streptococcus pyogenes & 5 & 7.14 \\
\hline Pseudomonas aeruginosa & 5 & 7.14 \\
\hline Escherichia coli & 5 & 7.14 \\
\hline Citrobacter koseri & 4 & 5.71 \\
\hline Enterobacter aerogenes & 3 & 4.28 \\
\hline Stenotrophomonas maltophila & 3 & 4.28 \\
\hline Acinetobacter baumannii & 3 & 4.28 \\
\hline Serratia marcescens & 2 & 2.85 \\
\hline Moraxella catarrhalis & 2 & 2.85 \\
\hline Burkholderia cepacia & 1 & 1.42 \\
\hline Achomobacter xylosoxidans & 1 & 1.42 \\
\hline TOTAL & 70 & $99.99 \%$ \\
\hline
\end{tabular}

The organisms obtained from the non acceptable category (4 of 42) included Staphylococcus aureus-2, Pseudomonas aeruginosa -1 and Escherichia coli-1. Yeasts in the present study were obtained only as a mixed growth, and hence were considered to be non-pathogenic. There was no multiple pathogen growth seen in the present study.

\section{Discussion}

Examination of expectorated sputum has been the primary means of determining the causes of bacterial pneumonia. However, lower respiratory tract secretions are always contaminated with upper tract flora present in the saliva. Hence, sputum is among the least clinically relevant specimens received for culture in the Microbiology laboratory, even though it is one of the most numerous and time consuming specimens. Good sputum samples depend on thorough healthcare worker education and patient understanding[4].The sputum grading system was initially given by Bartlett. This gives an indication whether the specimen represents the site of infection[5]. Out of 120 sputum samples processed during a one year period in the present study, 78 samples 
$(65 \%)$ could be considered in the acceptable category based on Bartlett's screening criteria used, and 42 samples $(35 \%)$ in the non acceptable category. Others studies reporting similar acceptability percentages include Anevlavis et al- 63\% and Mariraj et al- 79\%. While Daniel Musher et al had reported a low percentage of 31\% acceptability, Ravichandran et al had reported that all $74(100 \%)$ of their sputum samples so screened were in the non acceptable category, which contained only mixed flora not acceptable for culture. Grading system for sputum does not apply for lower respiratory tract infections by Legionella species, Mycobacterium tuberculosis, fungi and viruses. The importance of micro-organisms recovered from respiratory samples must always be evaluated in light of clinical information[3]. Total culture positivity in the present study was $61.66 \%(74 / 120)$.

Culture positivity reported in other studies include- Jean J Lloveras- 57\%, Daniel Musher et al- 79\%, Somporn et al- 40.95\%, Nawfal Ali Mubarak- 41.7\%, Aroma Oberoi et al- 32\% \& Nihan Ziyade et al- 44.7\%. On the contrary Ravichandran et al grew potential pathogens only in 5\% of their specimens processed. Out of the 78 acceptable specimens in the present study, potential pathogens were grown in 70 samples $(89.74 \%)$. While M R Shariatzadeh et al grew potential pathogens only in $33.7 \%$ of their acceptable samples, Mariraj et al grew in $63.2 \%$ of their acceptable samples. Among the 42 samples in the non acceptable category in the present study, pathogens were grown in only in $4(9.52 \%)$. Mariraj et al had reported 2 out of their 21 non acceptable samples growing potential pathogens $(9.5 \%)$. A useful quality assurance tool for sputum cultures is the comparison of Gram's stain and culture. If organisms seen in smear do not grow in culture, or if organisms that grow in moderate to heavy quantities are not seen in the smear, the smear should be re-evaluated. Gram's stain is a relatively in-sensitive method $\left(10^{5} \mathrm{CFU} / \mathrm{ml}\right.$ should be present to be visualized ), so small numbers of bacteria in culture may not well be visualized in the smear[3]. Gram's stain sensitivity in the present study (correlating Gram's character in direct smear and pathogen isolated in culture) among the acceptable category is 79.48\%. Other studies reporting include Daniel et al- 71.73\% and Nihan Ziyade et al- 78.6\%. Gram Negative Bacilli (GNB) were isolated in 48 cases (68.57\%) in the present study, and Gram Positive Cocci (GPC) in 22 (31.43\%) cases. Somporn et al had reported GNB in $76 \%$ of their isolates. The most common organisms isolated in the present study include: Klebsiella pneumoniae- $22.85 \%$, Streptococcus pneumoniae- $11.49 \%$ and Staphylococcus aureus- $10 \%$, which correlates well with other studies[1,6,8,9,10]. In a study by Ravichandran et al, the authors had concluded that sputum Gram's stain, sputum culture and blood cultures, in non severe CAP do not provide any diagnostically useful information and do not help in guiding for initial therapy. They concluded that the tests may be reserved for severe cases of CAP. In a study by Mariraj et al, the authors had concluded that Microbiology laboratories may reject for culture, those sputum samples which fail to meet the criteria of Bartlett for purulence, and sputum cultures must be ordered judiciously for documented episodes of LRTIs to provide a meaningful output. As authors of the present study, we completely agree with the conclusions of Mariraj et al, to reject such sputum samples which do not satisfy the Bartlett's criteria and order for fresh and proper samples. Criteria should be established by each laboratory for rejection of unsuitable specimens. Although general guidelines exist, director of each laboratory must decide which parameters to utilize. When a specimen is rejected, the person who submitted it should be made aware. Rejection criteria should be clearly listed in the laboratory service area[3]. Clinical relevance should be applied in diagnostic Microbiology. The point is to provide clinicians with information that will allow them to give best care to their patients[3].

\section{Conclusions}

A total of 120 sputum samples were processed during a one year period (Jan. to Dec. 2012). Based on Bartlett's screening procedure, $78(65 \%)$ were in the acceptable category and $42(35 \%)$ were in the nonacceptable category. Potential pathogen was grown in $70(89.74 \%)$ samples in the acceptable category, and 4 (9.52\%) samples in the non-acceptable category. Most common isolates obtained were Klebsiella pneumoniae$22.85 \%$, Streptococcus pneumoniae- $11.49 \%$ and Staphylococcus aureus- $10 \%$. As authors of the present study, we recommend initial screening of sputum samples for clinically relevant results, and reject the non-acceptable samples, and re-order for fresh and correct specimens.

\section{Acknowledgement}

We would like to thank Dr Gaurav Rastogi, Pathologist at SRL diagnostics, Hyderabad, with whose cooperation we could carry on the work successfully.

\section{References}

[1]. Nihan Ziyade, Aysegul Yagci. Improving sputum culture results for diagnosis of lower respiratory tract infections by saline washing. Marmara Medical Journal, 2010; 23(1): 30-36.

[2]. Ravichandran Theerthakarai, Walid El Halees, Medhat Ismail, Roberto A. Solis, M Anees Khan. Non value of Initial Microbiological Studies in the management of non severe Community Acquired Pneumonia. Chest, January 2001; 119(1): 181-184.

[3]. Washington Winn Jr, Stephen Allen, William Janda, Elmer Koneman. Guidelines for collection, transport, processing, analysis and reporting of cultures from specific specimen sources. In: Koneman's colour atlas and textbook of Microbiology, $6^{\text {th }}$ edition. Lippincott, Williams and Wilkins publications, 2006: 68-111. 
[4]. Fuselier PA, Garcin LS, Procop GW. Infections of the Lower Respiratory Tract. In: Betty AF, Daniel FS, Alice SW, editors. Bai ley and Scott's Diagnostic Microbiology. Mosby, 2002; 884-898..

[5]. Stavros Anevlavis, Niki Petroglon, Athanasios Tzavaras, Efstratios Maltzos, Ioannis Pneumatikos, Marios Froudarakis, Eleftherios Anevlavis, Demosthenes Bouros. A prospective study of the diagnostic utility of sputum Gram stain in pneumonia. Journal of Infection, 2009; 59: 83-89.

[6]. Mariraj J, Surekha Y, Asangi, Krishna S, Suresh B Sonth, Ramesh, Shanmugam. Sputum Gram's stain assessment in relation to sputum culture for Respiratory Tract Infections in a tertiary care hospital. Journal of Clinical and Diagnostic Research, December 2011; 5(8): 1699-1700.

[7]. Daniel M Musher, Roberto Montoya, Anna Wanahita. Diagnostic value of microscopic examination of Gram stained sputum and sputum cultures in patients with Pneumococcal pneumonia. Clinical Infectious Diseases, 2004; 39: 165-169.

[8]. Jean Jacques Lloveras, Mohamed -Issam, Shukr, Claude Pinos, Anissa Lindoulsi, Philippe Grima. Usefulness of sputum Gram's stain and culture for diagnosis of pneumonia in Geriatric institution. Journal of IMAB, 2010; 16(3): 20-22.

[9]. Nawfal Ali Mubarak. The findings of sputum culture of intubated mechanically ventilated patients versus non intubated patients in the Intensive Care Unit. Basrah Journal of Surgery, September 2012; 18: 1-5.

[10]. Aroma Oberoi, Aruna Aggarwal. Bacteriological profile, Serology and Antibiotic Sensitivity Pattern of Micro-organisms from Community Acquired Pneumonia. J K Science, April-June 2006; 8(2): 79-82.

[11]. M R Shariatzadeh \& T J Marrie. Does sputum culture affect the outcome of community acquired pneumonia? Eastern Mediterranean Health Journal, 2009; 15(4): 792-799.

[12]. Somporn Srifuengfung, Malinee Sangsawang, Podjanee Komolpis, Chertsak Dhiraputra and Busabawart Chompanee. Bacterial pathogens (non- Mycobacterial) from sputum culture and their anti-microbial susceptibility. March 1998; 29(1): 96-99.

[13]. M Rahman. Quality of specimens and sputum culture results: a retrospective study. Post-Graduate Medical Journal, August 1979; 55: $553-555$. 